\title{
WEAK SOLUTIONS TO THE TIME-FRACTIONAL G-NAVIER-STOKES EQUATIONS AND OPTIMAL CONTROL
}

\author{
SULTANA BEN AADI, KHALID AKHLIL, AND KHADIJA AAYADI
}

\begin{abstract}
In this paper we introduce the $g$-Navier-Stokes equations with time-fractional derivative of order $\alpha \in(0,1)$ in domains of $\mathbb{R}^{2}$. We then study the existence and uniqueness of weak solutions by means of Galerkin approximation. Finally, an optimal control problem is considered and solved.
\end{abstract}

\section{INTRODUCTION}

Hale and Raugel 9, 10, studied 3d nonlinear equations in thin domains of the form $\Omega_{\varepsilon}=\Omega \times(0, \varepsilon)$, where $\Omega \subset \mathbb{R}^{2}$ and $0<\varepsilon<1$. Afterwards Raugel and Sell [30] studied Navier-Stokes equations in thin domains. This developments found applications in Lake equations and Shallow water equations [7, 21, 22. J. Roh in his doctoral thesis [31, has employed the techniques developed by Hale, Raugel and Sell to the Navier-Stokes equations on thin domains of the form $\Omega_{g}=\Omega \times(0, g)$, where $g$ is some smooth scalar function. The derived equations are called the $g$-NavierStokes equations. The main difference between the classical Navier-Stokes equations and the $g$-Navier-Stokes equations is a weighted divergence condition of the form $\nabla \cdot(g u)=0$.

Much has already been done in the theory of $g$-Navier-Stokes equations. The existence of weak solutions is carried out in [6; see also [31, 32, 29. The stability and long-time behaviour questions can be found in [3, 4, 26, 5. Attractors of gNavier-Stokes equations attracted most of the interest of researchers 12, 13, 14, 15, [16, 17, 18, 27, 28, 33, 36, 37.

In this paper, we propose to study the weak solutions to time-fractional $g$-NavierStokes equations in the following form

$$
\left\{\begin{array}{rlrl}
\partial_{t}^{\alpha} u-\nu \Delta u+(u . \nabla) u+\nabla p & = & \text { in }(0, T) \times \Omega \\
\nabla \cdot(g u) & =0 & \text { in }(0, T) \times \Omega \\
u(0, .) & = & u_{0} & \text { in } \Omega
\end{array}\right.
$$

where where $\partial_{t}^{\alpha}$ is the Caputo fractional derivative of order $\alpha \in(0,1), u=u(x, t)=$ $\left(u_{1}, u_{2}\right)$ is the unknown velocity vector, $p=p(x, t)$ is the unknown pressure, $\nu>0$ is the kinematic viscosity coefficient, $u_{0}$ is the initial velocity, and $f$ represents the external force field.

The case where $\alpha=1$ and $g=1$ is just the standard Navier-Stokes equations and is a well-know subject, for more details, we refer to the monographs [25, 38, 34, 8, 20, 24] and references therein. The case where $\alpha=1$ and $g \neq 1$ is the $g$-Navier-Stokes equations as described above. The case where $0<\alpha<1$ and $g=1$ was considered first by Lions in $\left[23\right.$ but for order less than $\frac{1}{4}$ provided the

Date: January 8, 2021.

Key words and phrases. Matrix Operator,Fractional Evolution Equations. 
space dimension is not further than 4. In a recent work of Zhou and Peng [39, the question of weak solutions and optimal control problem of time-fractional NavierStokes equations is considered. In the current paper we will consider the case where $0<\alpha<1$ and $g \neq 1$. We will prove that problem 1.1 has a unique weak solution in domains $\Omega \subset \mathbb{R}^{2}$ with enough smooth boundaries.

As a second step, we will consider the following control problem associated with equations (1.1):

$$
\left\{\begin{aligned}
\partial_{t}^{\alpha} u-\nu \Delta u+(u \cdot \nabla) u+\nabla p & =C w+f \quad \text { in }(0, T) \times \Omega \\
\nabla \cdot(g u) & =0 \quad \text { in }(0, T) \times \Omega \\
u(0, .) & =u_{0} \quad \text { in } \Omega
\end{aligned}\right.
$$

where $w:[0, T] \rightarrow U_{g}$ and $C: U_{g} \rightarrow\left(L_{g}^{2}(\Omega)\right)^{2}$, with $U_{g}$ is some Hilbert space and $L_{g}^{2}(\Omega)$ is the standard Sobolev space with weight $g$.

This paper is organized as follows: In the first section, we will recall some concept and notations related to fractional calculus. Section 2, is devoted to the problem statement while section 3 will be dedicated to the proof of the existence and uniqueness of weak solutions to time-fractional g-Navier-Stokes equations. Finally section 4 will be concerned with the existence of an optimal control to (1.2).

\section{Preliminaries}

In this section, we provide some notations and preliminary results concerning fractional calculus. For this purpose, assume $X$ to be a Banach space. Let $\alpha \in(0,1]$ and let $k_{\alpha}$ denote the Riemann-Liouville kernel

$$
k_{\alpha}(t)=\frac{t^{\alpha-1}}{\Gamma(\alpha)}
$$

For a function $v:[0, T] \rightarrow X$, we give the following definitions of derivatives and integrals:

(1) The left Riemann-Liouville integral of $v$ is defined by

$$
{ }_{0} I_{t}^{\alpha} v(t)=\int_{0}^{t} k_{\alpha}(t-s) v(s) d s, \quad t>0
$$

provided the integral is point-wise defined on $[0,+\infty[$

(2) The right Riemann-Liouville integral of $v$ is defined by

$$
{ }_{t} I_{T}^{\alpha} v(t)=\int_{t}^{T} k_{\alpha}(t-s) v(s) d s, \quad t>0
$$

provided the integral is point-wise defined on $[0,+\infty[$

(3) The left Caputo fractional derivative of order $\alpha$ of $v$, is defined by

$$
{ }_{0}^{C} D_{t}^{\alpha} v(t)=\int_{0}^{t} k_{1-\alpha}(t-s) \frac{d}{d s} v(s) d s
$$

(4) The right Riemann-Liouville fractional derivative of order $\alpha$ of $v$ is defined by

$$
{ }_{t} D_{T}^{\alpha} v(t)=-\frac{d}{d t} \int_{t}^{T} k_{1-\alpha}(t-s) v(s) d s
$$


(5) The Liouville-Weyl fractional integral on the real axis for functions $v: \mathbb{R} \rightarrow$ $X$ is defined as follows

$$
{ }_{-\infty} I_{t}^{\alpha} v(t)=\int_{-\infty}^{t} k_{\alpha}(t-s) v(s) d s .
$$

(6) The Caputo fractional derivative on the real axis for functions $v: \mathbb{R} \rightarrow X$ is defined as follows

$$
{ }_{-\infty}^{C} D_{t}^{\alpha} v(t)={ }_{-\infty} I_{t}^{1-\alpha} \frac{d}{d t} v(t) .
$$

Note that the notation $\partial_{t}^{\alpha}$ stands for Caputo fractional partial derivative, i.e. when functions have another argument than time. We have the following fractional integration by parts formula; see, e.g. [1]

$$
\begin{aligned}
\int_{0}^{T}\left(\partial_{t}^{\alpha} u(t), \psi(t)\right) d t & =\int_{0}^{T}\left(u(t){ }_{t} D_{T}^{\alpha} \psi(t)\right) d t+\left.\left(u(t),_{t} I_{T}^{1-\alpha} \psi(t)\right)\right|_{0} ^{T} \\
& =\int_{0}^{T}\left(u(t){ }_{t} D_{T}^{\alpha} \psi(t)\right) d t-\left(u(0),{ }_{0} I_{T}^{1-\alpha} \psi(t)\right)
\end{aligned}
$$

since for $\psi \in C_{0}^{\infty}([0, T], X)$ one have $\lim _{t \rightarrow T}{ }_{t} I_{T}^{1-\alpha} \psi(t)=0$.

To pass from weak convergence to strong convergence we will need a compactness result. Let $X_{0}, X, X_{1}$ be Hilbert spaces with $X_{0} \hookrightarrow X \hookrightarrow X_{1}$ being continuous and $X_{0} \hookrightarrow X$ being compact. Assume that $v: \mathbb{R} \rightarrow X_{1}$ and denote by $\widehat{v}$ its Fourier transform

We have for $\gamma>0$

$$
\widehat{v}(\tau)=\int_{-\infty}^{+\infty} e^{-2 i \pi t \tau} v(t) d t
$$

$$
\widehat{{ }_{-\infty} D_{t}^{\gamma}} v(\tau)=(2 i \pi \tau)^{\gamma} \widehat{v}(\tau) .
$$

For a given $0<\gamma<1$, we introduce the following space

$$
W^{\gamma}\left(\mathbb{R}, X_{0}, X_{1}\right)=\left\{v \in L^{2}\left(\mathbb{R}, X_{0}\right):{ }_{-\infty}^{C} D_{t}^{\gamma} v \in L^{2}\left(\mathbb{R}, X_{1}\right)\right\} .
$$

Clearly, it is a Hilbert space for the norm

$$
\|v\|_{\gamma}=\left(\|v\|_{L^{2}\left(\mathbb{R}, X_{0}\right)}^{2}+\left\||\tau|^{\gamma} \widehat{v}\right\|_{L^{2}\left(\mathbb{R}, X_{1}\right)}^{2}\right)^{1 / 2} .
$$

For any set $K \subset \mathbb{R}$, we associate with it the subspace $W_{K}^{\gamma} \subset W^{\gamma}$ defined as

$$
W_{K}^{\gamma}\left(\mathbb{R}, X_{0}, X_{1}\right)=\left\{v \in W^{\gamma}\left(\mathbb{R}, X_{0}, X_{1}\right): \operatorname{support} u \subset K\right\} .
$$

By similar discussion as in the proof of Theorem 2.2 in Temam 35] (see also Theorem 2.1 in [39]), it is clear that $W_{K}^{\gamma}\left(\mathbb{R}, X_{0}, X_{1}\right) \hookrightarrow L^{2}(\mathbb{R}, X)$ is compact for any bounded set $K$ and any $\gamma>0$.

As a particular situation of the compactness result discussed above, let $H, V$ be two Hilbert spaces endowed with the scalar product $(.,)_{H}$ and $(., .)_{V}$ and the norms $|.|_{H}$ and $\|.\|_{V}$, respectively. Denote by $\langle.,$.$\rangle the dual pairing between V$ and $V^{\prime}$, the dual of $V$. Moreover assume that $V \hookrightarrow H \hookrightarrow V^{\prime}$ continuously and compactly and note that the space

$$
W^{\gamma}\left(0, T ; V, V^{\prime}\right)=\left\{v \in L^{2}(0, T ; V): \partial_{t}^{\gamma} v \in L^{2}\left(0, T ; V^{\prime}\right)\right\}
$$


is compactly embedded in $L^{2}(0, T ; H)$. Similarly to Lemma 2.1 in [39], we have

$$
\partial_{t}^{\gamma}(u(t), v)_{V}=\left\langle\partial_{t}^{\gamma} u(t), v\right\rangle
$$

for $u \in W^{\gamma}\left(0, T ; V, V^{\prime}\right)$ and $v \in H$. Moreover, for a differentiable function $v$ : $[0, T] \rightarrow V$ we have from 2 that

$$
\left(v(t),{ }_{0}^{C} D_{t}^{\gamma} v(t)\right)_{H} \geq \frac{1}{2}{ }_{0}^{C} D_{t}^{\gamma}|v(t)|^{2} .
$$

We end this section by the following important result

Lemma 2.1. Suppose that a nonnegative function satisfies

$$
{ }_{0}^{C} D_{t}^{\gamma} v(t)+c_{1} v(t) \leq c_{2}(t)
$$

for $c_{1}>0$ and $c_{2}$ a nonnegative integrable function for $t \in[0, T]$. Then

$$
v(t) \leq v(0)+\frac{1}{\Gamma(\gamma)} \int_{0}^{t}(t-s)^{\gamma-1} v(s) d s
$$

For more details about fractional calculus we refer to the monographs [19, 40, 41].

\section{Problem Statement}

Let $\Omega$ be a bounded domain of $\mathbb{R}^{2}$ with smooth boundary. Let $g \in W^{1, \infty}(\Omega)$ such that $0<m_{0}<g(x)<M_{0}$ for all $x \in \Omega$. To deal with the weighted divergence condition $\nabla \cdot g u=0$ we rewrite the Navier-Stokes equations in weighted Sobolev spaces. This bring no further difficulties thanks to the definition of $g$.

Let $L_{g}^{2}(\Omega)=\left(L^{2}(\Omega)\right)^{2}$ and $H_{g}^{1}(\Omega)=\left(H^{1}(\Omega)\right)^{2}$ be endowed, respectively, with the inner products

$$
\begin{gathered}
(u, v)_{g}=\int_{\Omega} u . v g d x, \quad u, v \in L_{g}^{2}(\Omega) \\
((u, v))_{g}=\int_{\Omega} \sum_{j=1}^{2} \nabla u_{j} . \nabla v_{j} g d x, \quad u, v \in H_{g}^{1}(\Omega)
\end{gathered}
$$

and norms $|\cdot|_{g}^{2}=(., .)_{g},\|\cdot\|_{g}^{2}=((., .))_{g}$. Note that the norms $|\cdot|_{g}$ and $\|\cdot\|_{g}$ are equivalent to the usual norms in $\left(L^{2}(\Omega)\right)^{2}$ and $\left(H^{1}(\Omega)\right)^{2}$.

Let us introduce the following spaces

$$
\begin{aligned}
V & =\left\{u \in\left(C_{0}^{\infty}(\Omega)\right)^{2}: \nabla \cdot(g u)=0 \text { in } \Omega\right\}, \\
H_{g} & =\text { the closure of } V \text { in } L_{g}^{2}(\Omega), \\
V_{g} & =\text { the closure of } V \text { in } H_{g}^{1}(\Omega) .
\end{aligned}
$$

It follows that

$$
V_{g} \subset H_{g} \simeq H_{g}^{\prime} \subset V_{g}^{\prime}
$$

where the injections are dense and continuous. We denote by $\|\cdot\|_{*}$ the norm in $V_{g}^{\prime}$ and by $\langle.,$.$\rangle the duality pairing between V_{g}$ and its dual $V_{g}^{\prime}$.

Set $A_{g}: V_{g} \rightarrow V_{g}^{\prime}$ defined by $\left\langle A_{g} u, v\right\rangle=((u, v))_{g}$. Denote $D\left(A_{g}\right)=\left\{u \in V_{g}\right.$ : $\left.A_{g} u \in H_{g}\right\}$. Then $D\left(A_{g}\right)=H_{g}^{2}(\Omega) \cap V_{g}$ and $A_{g} u=P_{g} \Delta u$ for all $u \in D\left(A_{g}\right)$ where $P_{g}$ is the orthogonal projection from $L_{g}^{2}(\Omega)$ onto $H_{g}$. 
Set $B_{g}: V_{g} \times V_{g} \rightarrow V_{g}^{\prime}$ defined by $\langle B(u, v), w\rangle=b_{g}(u, v, w)$, where

$$
b_{g}(u, v, w)=\sum_{j, k=1}^{d} \int_{\Omega} u_{j} \frac{\partial v_{k}}{\partial x_{j}} w_{k} g d x
$$

whenever the integral make sense. It is easy to check that if $u, v, w \in V_{g}$ then $b_{g}(u, v, w)=-b_{g}(u, w, v)$. Hence

$$
b_{g}(u, v, v)=0, \quad \forall u, v \in V_{g} .
$$

Let $u \in L^{2}\left(0, T ; V_{g}\right)$, then $C_{g} u$ defined by

$$
\left(C_{g} u(t), v\right)_{g}=\left(\left(\frac{\nabla g}{g} \cdot \nabla\right) u, v\right)_{g}=b_{g}\left(\frac{\nabla g}{g}, u, v\right), \quad \forall v \in V_{g}
$$

Since

we have

$$
-\frac{1}{g}(\nabla \cdot g \nabla) u=-\Delta u-\left(\frac{\nabla g}{g} \cdot \nabla\right) u
$$

$$
\begin{aligned}
(-\Delta u, v)_{g} & =((u, v))_{g}+\left(\left(\frac{\nabla g}{g} \cdot \nabla\right) u, v\right)_{g} \\
& =\left(A_{g} u, v\right)_{g}+\left(\left(\frac{\nabla g}{g} \cdot \nabla\right) u, v\right)_{g} \quad \forall u, v \in V_{g} .
\end{aligned}
$$

Proposition 3.1 (31]). For the g-Stokes operator $A_{g}$, the following hold:

(1) The g-Stokes operator $A_{g}$ is positive, self-adjoint with compact inverse, where the domain of $A_{g}$ is $D\left(A_{g}\right)=V_{g} \cap H_{g}^{2}(\Omega)$.

(2) There exists countably many eigenvalues of $A_{g}$ satisfying

$$
0<\frac{4 \pi^{2} m_{0}}{M_{0}} \leq \lambda_{1} \leq \lambda_{2} \leq \ldots
$$

where $\lambda_{1}$ is the smallest eigenvalue of $A_{g}$. In addition, there exists the corresponding collection of eigenfunctions $\left\{u_{i}\right\}_{i \in \mathbb{N}}$ forming an orthonormal basis for $H_{g}$.

Since the operators $A_{g}$ and $P_{g}$ are self-adjoint, using integration by parts, we have

$$
\left(A_{g} u, u\right)_{g}=\left(P_{g}(-\Delta u), u\right)_{g}=\int_{\Omega} \nabla u . \nabla u g d x=|\nabla u|_{g}^{2}
$$

and $b_{g}$ satisfies

$$
\left|b_{g}(u, v, w)\right|_{g} \leq c|u|_{g}^{\frac{1}{2}}\|u\|_{g}^{\frac{1}{2}}|v|_{g}^{\frac{1}{2}}|w|_{g}^{\frac{1}{2}}\|w\|_{g}^{\frac{1}{2}}, \quad \forall u, v, w \in V_{g} .
$$

Denote the operator $C_{g} u=P_{g}\left(\frac{1}{g}(\nabla g . \nabla) u\right)$ such that $\left(C_{g} u, v\right)_{g}=b_{g}\left(\frac{\nabla g}{g}, u, v\right)$ and the operator $B_{g}[]=.B(.,$.$) .$

Definition 3.2. Let $f \in L^{2 / \alpha_{1}}\left(0, T ; V_{g}^{\prime}\right)$ for some $\alpha_{1} \in(0, \alpha)$ and $u_{0} \in H_{g}$ be given. A function $u \in L^{2}\left(0, T ; V_{g}\right)$ with $u_{t} \in L^{2}\left(0, T ; V_{g}^{\prime}\right)$ is called a weak solution of problem (1.1) if it fulfils

$$
\left\{\begin{aligned}
\partial_{t}^{\alpha} u+\nu A_{g} u+\nu C_{g} u+B_{g}[u] & =f \quad \text { in } L^{2}\left(0, T ; V_{g}^{\prime}\right) \\
u(0) & =u_{0}, \quad \text { in } H_{g}
\end{aligned}\right.
$$




\section{Existence And Uniqueness}

In this section we prove the existence and uniqueness of the weak solution of problem (3.1) under the following hypothesis:

$$
H(g): \quad|\nabla g|_{\infty}<\frac{1}{2} m_{0} \lambda_{1}^{\frac{1}{2}}
$$

where $\lambda_{1}$ is defined in Proposition 3.1. We will apply Faedo-Galerkin Method as initially used in 35. for classical Navier-Sokes equations and in 41] for timefractional version of it.

Theorem 4.1. Let $\Omega$ be a bounded and locally Lipschitz domain in $\mathbb{R}^{2}$. Let $f \in$ $L^{2}\left(0, T ; V_{g}^{\prime}\right)$ and $u_{0} \in H_{g}$. Then, under the hypothesis $H(g)$, the equation (3.1) has a unique weak solution $u \in L^{2}\left(0, T ; V_{g}\right) \cap L^{\infty}\left(0, T ; H_{g}\right)$.

Proof. We apply Faedo-Galerkin Method. Since $V_{g}$ is separable, there exists a sequence $\left\{u_{i}\right\}_{i \in \mathbb{N}}$ which forms a complete orthonormal system in $H_{g}$ and a basis for $V_{g}$. Let $m$ be a positive integer. For each $m$, we define an approximate solution $u^{(m)}(t)$ of (3.1) as a solution of the system

$$
\left\{\begin{array}{r}
\left({ }_{0}^{C} \mathrm{D}_{t}^{\alpha} u^{(m)}, u_{k}\right)_{g}+\nu\left(\left(u^{(m)}, u_{k}\right)\right)_{g}+\nu b_{g}\left(\frac{\nabla g}{g}, u^{(m)}, u_{k}\right)+b_{g}\left(u^{(m)}, u^{(m)}, u_{k}\right)=\left(f, u_{k}\right)_{g} \\
\left(u^{(m)}(0), u_{k}\right)_{g}=\left(u_{0}, u_{k}\right)_{g} .
\end{array}\right.
$$

Let $\xi_{k}=\xi_{k}(t)$ denote the $k$ th component of $u^{(m)}(t)$, i.e. $\xi_{k}(t)=\left(u^{(m)}(t), u_{k}\right)_{g}$. Also, let $\eta_{k}(t)=\left(f(t), u_{k}\right)_{g}$ be component of $f(t)$. Then (4.1) is equivalent to a nonlinear fractional order ordinary differential equation for the functions $\xi_{k}$

$$
\left\{\begin{aligned}
&{ }_{0}^{C} \mathrm{D}_{t}^{\alpha} \xi_{k}+\nu \lambda_{k} \xi_{k}+\nu \sum_{l=1}^{m} b_{g}\left(\frac{\nabla g}{g}, u_{l}, u_{k}\right) \xi_{l} \\
&+\sum_{l, l^{\prime}=1}^{m} b_{g}\left(u_{l}, u_{l^{\prime}}, u_{k}\right) \xi_{l} \xi_{l^{\prime}}=\eta_{k} \quad, k=1, \ldots, m \\
& \xi_{k}(0)=\xi_{k}^{0} .
\end{aligned}\right.
$$

The system forms a nonlinear first order system of fractional ordinary differential equation for the functions $\xi_{k}(t)$ and has maximal solutions on some interval $\left[0, t_{m}\right]$. If $t_{m}<T$, then $|\xi(t)|$ must go to $+\infty$ as $t \rightarrow t_{m}$, where $\xi=\left(\xi_{1}, \xi_{2}, \ldots, \xi_{n}\right)$ and |.| the euclidean norm in $\mathbb{R}^{n}$. We denote $\eta=\left(\eta_{1}, \eta_{2}, \ldots, \eta_{n}\right)$ and $(.,$.$) the usual$ euclidean scalar product in $\mathbb{R}^{n}$, one obtains, from (4.2), that

$$
\left({ }_{0}^{C} \mathrm{D}_{t}^{\alpha} \xi(t), \xi(t)\right)+\nu \sum_{k=1}^{m} \lambda_{k} \xi_{k}^{2}+\nu \sum_{k, l=1}^{m} b_{g}\left(\frac{\nabla g}{g}, u_{l}, u_{k}\right) \xi_{l} \xi_{k}=(\eta, \xi)
$$

because $\sum_{k, l, l^{\prime}=1}^{m} b_{g}\left(u_{l}, u_{l^{\prime}}, u_{k}\right) \xi_{l} \xi_{l^{\prime}} \xi_{k}=0$. It then follows that 


$$
\begin{aligned}
\frac{1}{2}{ }_{0}^{C} \mathrm{D}_{t}^{\alpha}|\xi(t)|^{2}+\nu \sum_{k=1}^{m} \lambda_{k} \xi_{k}^{2} & \leq(\eta, \xi)-\nu \sum_{k, l=1}^{m} b_{g}\left(\frac{\nabla g}{g}, u_{l}, u_{k}\right) \xi_{l} \xi_{k} \\
& \leq\left(\sum_{k=1}^{m} \lambda_{k}^{-1} \eta_{k}^{2}\right)^{\frac{1}{2}}\left(\sum_{k=1}^{m} \lambda_{k} \xi_{k}^{2}\right)^{\frac{1}{2}}+\frac{\nu|\nabla g|_{\infty}}{m_{0}} \sum_{k=1}^{m}\left|u_{k}\right|\left|\nabla u_{k}\right| \\
& \leq \frac{1}{2 \lambda_{1} \nu}|\eta(t)|^{2}+\frac{\nu}{2} \sum_{k=1}^{m} \lambda_{k} \xi_{k}^{2}+\frac{\nu|\nabla g|_{\infty}}{m_{0} \lambda_{1}^{\frac{1}{2}}} \sum_{k=1}^{m} \lambda_{k} \xi_{k}^{2} .
\end{aligned}
$$

It follows that

$$
{ }_{0}^{C} \mathrm{D}_{t}^{\alpha}|\xi(t)|^{2}+\nu^{\prime} \sum_{k=1}^{m} \lambda_{k} \xi_{k}^{2} \leq \frac{1}{\lambda_{1} \nu}|\eta(t)|^{2}
$$

where $\nu^{\prime}=\nu\left(1-\frac{2|\nabla g|_{\infty}}{m_{0} \lambda_{1}^{\frac{1}{2}}}\right)>0$ (because of $\left.H(g)\right)$. It follows that

$$
{ }_{0}^{C} \mathrm{D}_{t}^{\alpha}|\xi(t)|^{2}+\nu^{\prime} \lambda_{1}|\xi(t)|^{2} \leq \frac{1}{\lambda_{1} \nu}|\eta(t)|^{2} .
$$

We have the estimates

$$
\begin{aligned}
|\xi(t)|^{2} & \leq|\xi(0)|^{2}+\frac{1}{\nu^{\prime} \lambda_{1} \Gamma(\alpha)} \int_{0}^{t}(t-s)^{\alpha-1}|\eta(s)|^{2} d s \\
& \leq|\xi(0)|^{2}+\frac{1}{\nu^{\prime} \lambda_{1} \Gamma(\alpha)} \int_{0}^{t}|\eta(s)|^{2 / \alpha_{1}} d s+\frac{1}{\nu^{\prime} \lambda_{1} \Gamma(\alpha)} \int_{0}^{t}(t-s)^{\frac{\alpha-1}{1-\alpha_{1}}} d s \\
& \leq|\xi(0)|^{2}+\frac{1}{\nu^{\prime} \lambda_{1} \Gamma(\alpha)} \int_{0}^{t}|\eta(s)|^{2 / \alpha_{1}} d s+\frac{T^{1+b}}{(1+b) \nu^{\prime} \lambda_{1} \Gamma(\alpha)} \\
& \leq M
\end{aligned}
$$

where $\alpha_{1} \in(0, \alpha), b=\frac{\alpha-\alpha_{1}}{1-\alpha_{1}}$. Therefore $t_{m}=T$.

We multiply (4.1) by $\xi_{k}(t)$ and sum this equations for $k=1, \ldots, m$. Taking into account that $b_{g}\left(u^{(m)}, u^{(m)}, u^{(m)}\right)=0$, we get

$$
\left({ }_{0}^{C} \mathrm{D}_{t}^{\alpha} u^{(m)}, u^{(m)}\right)_{g}+\nu\left\|u^{(m)}(t)\right\|_{g}^{2}+\nu b_{g}\left(\frac{\nabla g}{g}, u^{(m)}(t), u^{(m)}(t)\right)=\left(f, u^{(m)}(t)\right)_{g} .
$$

Using Schwartz and Young inequalities, we get

$$
\begin{aligned}
\frac{1}{2}{ }_{0}^{C} \mathrm{D}_{t}^{\alpha}\left|u^{(m)}(t)\right|^{2}+\nu\left\|u^{(m)}(t)\right\|_{g}^{2} & \leq \frac{1}{2 \nu}\|f(t)\|_{V_{g}^{\prime}}^{2}+\frac{\nu}{2}\left\|u^{(m)}(t)\right\|_{g}^{2}+\frac{\nu|\nabla g|_{\infty}^{2}}{m_{0}^{2}}\left|u^{(m)}(t)\right|_{g}^{2} \\
& \leq \frac{1}{2 \nu}\|f(t)\|_{V_{g}^{\prime}}^{2}+\frac{\nu}{2}\left\|u^{(m)}(t)\right\|_{g}^{2}+\frac{\nu|\nabla g|_{\infty}^{2}}{\lambda_{1} m_{0}^{2}}\left\|u^{(m)}(t)\right\|_{g}^{2} .
\end{aligned}
$$

It follows that

$$
{ }_{0}^{C} \mathrm{D}_{t}^{\alpha}\left|u^{(m)}(t)\right|_{g}^{2}+\nu^{\prime}\left\|u^{(m)}(t)\right\|_{g}^{2} \leq \frac{1}{\nu}\|f(t)\|_{V_{g}^{\prime}}^{2}
$$

where $\nu^{\prime}=\nu\left(1-\frac{2|\nabla g|_{\infty}^{2}}{\lambda_{1} m_{0}^{2}}\right)$. Integrating (with order $\alpha$ ) we obtain 


$$
\begin{aligned}
\left|u^{(m)}(t)\right|_{g}^{2}+\nu^{\prime} \int_{0}^{t}(t-s)^{\alpha-1}\left\|u^{(m)}(s)\right\|_{g}^{2} d s & \leq\left|u_{0 m}\right|_{g}^{2}+\frac{1}{\nu^{\prime}} \int_{0}^{t}(t-s)^{\alpha-1}\|f(s)\|_{V_{g}^{\prime}}^{2} d s \\
& \leq\left|u_{0 m}\right|_{g}^{2}+\frac{1}{\nu^{\prime}} \int_{0}^{t}\|f(s)\|_{V_{g}^{\prime}}^{2 / \alpha_{1}} d s+\frac{1}{\nu^{\prime}} \int_{0}^{t}(t-s)^{\frac{\alpha-1}{1-\alpha_{1}}} d s \\
& \leq\left|u_{0 m}\right|_{g}^{2}+\frac{1}{\nu^{\prime}} \int_{0}^{t}\|f(s)\|_{V_{g}^{\prime}}^{2 / \alpha_{1}} d s+\frac{T^{1+b}}{(1+b) \nu^{\prime}} .
\end{aligned}
$$

It follows that for almost every $t \in[0, T]$

$$
\begin{gathered}
\sup _{t \in[0, T]}\left|u^{(m)}(t)\right|_{g}^{2} \leq\left|u_{0 m}\right|_{g}^{2}+\frac{1}{\nu^{\prime}} \int_{0}^{T}\|f(s)\|_{V_{g}^{\prime}}^{2 / \alpha_{1}} d s+\frac{T^{1+b}}{(1+b) \nu^{\prime}} \\
\int_{0}^{t}(t-s)^{\alpha-1}\left\|u^{(m)}(s)\right\|_{g}^{2} d s \leq \frac{1}{\nu^{\prime}}\left|u_{0 m}\right|_{g}^{2}+\frac{1}{\nu^{\prime 2}} \int_{0}^{t}\|f(s)\|_{V_{g}^{\prime}}^{2 / \alpha_{1}} d s+\frac{T^{1+b}}{(1+b) \nu^{\prime 2}} .
\end{gathered}
$$

The relation (4.3) ensures that the sequence $\left\{u^{(m)}\right\}_{m}$ is bounded in $L^{\infty}\left(0, T ; H_{g}\right)$. Moreover, from (4.4), one gets

$$
\begin{aligned}
T^{\alpha-1} \int_{0}^{t}\left\|u^{(m)}(s)\right\|_{g}^{2} d s & \leq \int_{0}^{t}(t-s)^{\alpha-1}\left\|u^{(m)}(s)\right\|_{g}^{2} d s \\
& \leq \frac{1}{\nu^{\prime}}\left|u_{0 m}\right|_{g}^{2}+\frac{1}{\nu^{\prime 2}} \int_{0}^{T}\|f(s)\|_{V_{g}^{\prime}}^{2 / \alpha_{1}} d s+\frac{T^{1+b}}{(1+b) \nu^{\prime 2}} .
\end{aligned}
$$

Hence the sequence $\left\{u^{(m)}\right\}_{m}$ is bounded in $L^{2}\left(0, T ; V_{g}\right)$.

Let $\tilde{u}^{(m)}: \mathbb{R} \rightarrow V_{g}$ denote the function defined by

$$
\tilde{u}^{(m)}(t)=\left\{\begin{array}{rr}
u^{(m)}(t), & 0 \leq t \leq T \\
0, & \text { otherwise }
\end{array}\right.
$$

and $\hat{u}^{(m)}$ denotes the Fourier transform of $\tilde{u}^{(m)}$. We show that the sequence $\left\{\tilde{u}^{(m)}\right\}_{m}$ remains bounded in $W^{\gamma}\left(\mathbb{R}, V_{g}, H_{g}\right)$. To do so, we need to verify that

$$
\int_{-\infty}^{+\infty}|\tau|^{2 \gamma}\left|\hat{u}^{(m)}(\tau)\right|^{2} d \tau \leq \text { const. for some } \gamma>0
$$

In order to prove (4.5), we observe that

$$
\left({ }_{0}^{C} D_{t}^{\alpha} \tilde{u}^{(m)}, u_{k}\right)_{g}=\left(\widetilde{F}_{m}, u_{k}\right)_{g}+\left(u_{m 0}, u_{k}\right)_{g-\infty} I_{t}^{1-\alpha} \delta_{0}-\left(u^{(m)}(T), u_{k}\right)_{g-\infty} I_{t}^{1-\alpha} \delta_{T}
$$

where $F_{m}=f-\nu A_{g} u^{(m)}-B_{g}\left(u^{(m)}, u^{(m)}\right)-\nu C_{g} u^{(m)}$ and $\delta_{0}, \delta_{T}$ are Dirac distributions at 0 and $T$. Here $\widetilde{F}_{m}$ is defined as usual by

$$
\widetilde{F}_{m}(t)=\left\{\begin{array}{rr}
F_{m}(t), & 0 \leq t \leq T \\
0, & \text { otherwise }
\end{array}\right.
$$

Indeed, it is classical that since $\tilde{u}^{(m)}$ has two discontinuities at 0 and $T$, the Caputo derivative of $\tilde{u}^{(m)}$ is given by 


$$
\begin{aligned}
{ }_{-\infty}^{C} D_{t}^{\alpha} \tilde{u}^{(m)} & ={ }_{-\infty} I_{t}^{1-\alpha}\left(\frac{d}{d t} \tilde{u}^{(m)}\right) \\
& ={ }_{-\infty} I_{t}^{1-\alpha}\left(\frac{d}{d t} u^{(m)}+u^{(m)}(0) \delta_{0}-u^{(m)}(T) \delta_{T}\right) \\
& ={ }_{0}^{C} D_{t}^{\alpha} u^{(m)}+{ }_{-\infty} I_{t}^{1-\alpha}\left(u^{(m)}(0) \delta_{0}-u^{(m)}(T) \delta_{T}\right) .
\end{aligned}
$$

By the Fourier transform (4.6) yields to

$$
\begin{aligned}
(2 i \pi \tau)^{\alpha}\left(\hat{u}^{(m)}, u_{k}\right)_{g}=\left(\widehat{F}_{m}, u_{k}\right)_{g} & +\left(u_{m 0}, u_{k}\right)_{g}(2 i \pi \tau)^{\alpha-1} \\
& -\left(u^{(m)}(T), u_{k}\right)_{g}(2 i \pi \tau)^{\alpha-1} e^{-2 i \pi T \tau}
\end{aligned}
$$

here $\hat{u}^{(m)}$ and $\widehat{F}_{m}$ denote the Fourier transforms of $\tilde{u}^{(m)}$ and $\widetilde{F}_{m}$, respectively.

We multiply by $\hat{\xi}_{k}(\tau)$ and sum these equations for $k=1, \ldots, m$ to get

$$
\begin{aligned}
(2 i \pi \tau)^{\alpha}\left|\hat{u}^{(m)}(\tau)\right|_{g}^{2}=\left(\widehat{F}_{m}(\tau), \hat{u}^{(m)}(\tau)\right)_{g}+\left(u_{m 0}, \hat{u}^{(m)}(\tau)\right)_{g}(2 i \pi \tau)^{\alpha-1} & \\
& -\left(u^{(m)}(T), \hat{u}^{(m)}(\tau)\right)_{g}(2 i \pi \tau)^{\alpha-1} e^{-2 i \pi T \tau} .
\end{aligned}
$$

We have

$$
\begin{gathered}
\int_{0}^{T}\left\|F_{m}(t)\right\|_{V_{g}^{\prime}} d t \leq c \int_{0}^{T}\left(\|f(t)\|_{V_{g}^{\prime}}+\left|u^{(m)}(t)\right|_{g}\left\|u^{(m)}(t)\right\|_{g}+\left\|u^{(m)}(t)\right\|_{g}\right. \\
\left.+|\nabla g|_{\infty}\left\|u^{(m)}(t)\right\|_{g}\right) d t .
\end{gathered}
$$

Therefore $\left\|F_{m}(t)\right\|_{V_{g}^{\prime}}$ is bounded in $L^{1}\left(0, T ; V_{g}^{\prime}\right)$. Hence

$$
\sup _{\tau \in \mathbb{R}}\left\|\widehat{F}_{m}(\tau)\right\|_{V_{g}^{\prime}} \leq c, \quad \forall m .
$$

Moreover, since $u_{m 0}$ and $u^{(m)}(T)$ are bounded we have

$$
\begin{aligned}
|\tau|^{\alpha}\left|\hat{u}^{(m)}(\tau)\right|_{g}^{2} & \leq c_{2}\left\|\hat{u}^{(m)}(\tau)\right\|_{g}+c_{3}|\tau|^{\alpha-1}\left|\hat{u}^{(m)}(\tau)\right|_{g} \\
& \leq c_{4}\left(1 \vee|\tau|^{\alpha-1}\right)\left\|\hat{u}^{(m)}(\tau)\right\|_{g} .
\end{aligned}
$$

For $\gamma$ fixed, $\gamma<\alpha / 4$, we observe that

$$
|\tau|^{2 \gamma} \leq c(\gamma) \frac{1+|\tau|^{\alpha}}{1+|\tau|^{\alpha-2 \gamma}}
$$

Then we can write

$$
\begin{aligned}
\int_{-\infty}^{+\infty}|\tau|^{2 \gamma}\left|\hat{u}^{(m)}(\tau)\right|_{g}^{2} & \leq c_{5}(\gamma) \int_{-\infty}^{+\infty} \frac{1+|\tau|^{\alpha}}{1+|\tau|^{\alpha-2 \gamma}}\left|\hat{u}^{(m)}(\tau)\right|_{g}^{2} d \tau \\
& \leq c_{6}(\gamma) \int_{-\infty}^{+\infty} \frac{1}{1+|\tau|^{\alpha-2 \gamma}}\left\|\hat{u}^{(m)}(\tau)\right\|_{g}^{2} d \tau \\
& \quad+c_{7}(\gamma) \int_{-\infty}^{+\infty} \frac{|\tau|^{\alpha-1}}{1+|\tau|^{\alpha-2 \gamma}}\left\|\hat{u}^{(m)}(\tau)\right\|_{g}^{2} d \tau
\end{aligned}
$$


By Parseval inequality, the first integral is bounded as $m \rightarrow \infty$. Applying the Schwartz inequality, the second integral yields to

$$
\begin{aligned}
\int_{-\infty}^{+\infty} \frac{|\tau|^{\alpha-1}}{1+|\tau|^{\alpha-2 \gamma}}\left\|\hat{u}^{(m)}(\tau)\right\|_{g}^{2} d \tau \leq\left(\int_{-\infty}^{+\infty} \frac{d \tau}{\left(1+|\tau|^{\alpha-2 \gamma}\right)^{2}}\right)^{1 / 2} \\
\times\left(\int_{-\infty}^{+\infty}|\tau|^{2 \alpha-2}\left\|\hat{u}^{(m)}(\tau)\right\|_{g}^{2} d \tau\right)^{1 / 2} .
\end{aligned}
$$

The first integral is finite due to $\gamma<\alpha / 4$. On the other hand, it follows from the Parseval equality that

$$
\begin{aligned}
\int_{-\infty}^{+\infty}|\tau|^{2 \alpha-2}\left\|\hat{u}^{(m)}(\tau)\right\|_{g}^{2} d \tau & =\int_{-\infty}^{+\infty}\left\|-\infty \mathrm{I}_{t}^{1-\alpha} \tilde{u}^{(m)}(t)\right\|_{g}^{2} d t \\
& =\int_{0}^{T}\left\|_{0} \mathrm{I}_{t}^{1-\alpha} u^{(m)}(t)\right\|_{g}^{2} d t \\
& \leq\left(\frac{T^{1-\alpha}}{\Gamma(2-\alpha)}\right)^{2} \int_{0}^{T}\left\|u^{(m)}(t)\right\|_{V}^{2} d t .
\end{aligned}
$$

Which implies that (4.5) holds.

We know that a subsequence of $\left\{u^{(m)}\right\}_{m}$ (which we will denote with the same symbol) converges to some $u$ weakly in $L^{2}\left(0, T ; V_{g}\right)$ and weak-star in $L^{\infty}\left(0, T ; H_{g}\right)$ with $u \in L^{2}\left(0, T ; V_{g}\right) \cap L^{\infty}\left(0, T ; H_{g}\right)$. As $W^{\gamma}\left(0, T, V_{g} ; H_{g}\right)$ is compactly embedded in $L^{2}\left(0, T ; H_{g}\right)$ then $\left\{u^{(m)}\right\}_{m}$ strongly converges in $L^{2}\left(0, T ; H_{g}\right)$.

In order to pass to the limit, we consider the scalar function $\psi$ continuously differentiable on $[0, T]$ and such that $\psi(T)=0$. In the first equation of the system (4.1), we consider $\psi(t) u_{k}$ instead of $u_{k}$ and then we integrate by parts. This leads to the equation

$$
\begin{gathered}
\int_{0}^{T}\left(u^{(m)}(t){ }_{,} \mathrm{D}_{T}^{\alpha} \psi(t) u_{k}\right)_{g} d t+\int_{0}^{T} b_{g}\left(u^{(m)}(t), u^{(m)}(t), \psi u_{k}\right) d t+\nu \int_{0}^{T}\left(\left(u^{(m)}(t), \psi u_{k}\right)\right)_{g} \\
\quad+\nu \int_{0}^{T} b_{g}\left(\frac{\nabla g}{g}, u^{(m)}(t), \psi u_{k}\right) d t=\left(u_{0 m}, 0 \mathrm{I}_{T}^{1-\alpha} \psi(t) u_{k}\right)_{g}+\int_{0}^{T}\left(f(t), u_{k}\right)_{g} d t .
\end{gathered}
$$

Moreover we have the following convergence

$$
\begin{aligned}
\lim _{m \rightarrow+\infty} \int_{0}^{T} b_{g}\left(u^{(m)}(t), u^{(m)}(t),\right. & \left.\psi(t) u_{k}\right) d t=-\lim _{m \rightarrow+\infty} \int_{0}^{T} b_{g}\left(u^{(m)}(t), \psi u_{k}, u^{(m)}(t)\right) d t \\
= & -\lim _{m \rightarrow+\infty} \sum_{i, j=1}^{d} \int_{0}^{T} \int_{\Omega} u_{i}^{(m)} \mathrm{D}_{i}\left(u_{k}\right)_{j} u_{j}^{(m)} d x \psi(t) g d x d t \\
& =-\sum_{i, j=1}^{d} \int_{0}^{T} \int_{\Omega}(u)_{i} \mathrm{D}_{i}\left(u_{k}\right)_{j}(u)_{j} d x \psi(t) g d x d t \\
& =-\int_{0}^{T} b_{g}\left(u(t), \psi u_{k}, u(t)\right) d t \\
& =\int_{0}^{T} b_{g}\left(u(t), u, \psi(t) u_{k}\right) d t .
\end{aligned}
$$


where $D_{i}$ stands for the partial derivative with respect to $x_{i}$. In the same way, one can prove that

$$
\lim _{m \rightarrow+\infty} \int_{0}^{T} b_{g}\left(\frac{\nabla g}{g}, u^{(m)}(t), \psi u_{k}\right) d t=\int_{0}^{T} b_{g}\left(\frac{\nabla g}{g}, u(t), \psi u_{k}\right) d t .
$$

It then follows that

$$
\begin{gathered}
\int_{0}^{T}\left(u(t){ }_{t} \mathrm{D}_{T}^{\alpha} \psi(t) u_{k}\right)_{g} d t+\int_{0}^{T} b_{g}\left(u(t), u(t), \psi u_{k}\right) d t+\nu \int_{0}^{T}\left(\left(u(t), \psi u_{k}\right)\right)_{g} \\
\quad+\nu \int_{0}^{T} b_{g}\left(\frac{\nabla g}{g}, u(t), \psi u_{k}\right) d t=\left(u_{0},{ }_{0} \mathrm{I}_{T}^{1-\alpha} \psi(t) u_{k}\right)_{g}+\int_{0}^{T}\left(f(t), u_{k}\right)_{g} d t .
\end{gathered}
$$

This equation holds for $v$ which is finite linear combination of $u_{k}, k=1, \ldots, m$ and by continuity it holds for any $v$ in $V_{g}$. It then follows that $u$ satisfies the equation (3.1). To end the proof it still to check that $u$ satisfies the initial condition $u(0)=u_{0}$. To do so it suffices to multiply (3.1) by $\psi$ and integrate. By making use of the integration by parts and comparing with (4.7), one can find that

$$
\left(u_{0}-u(0), v\right)_{g} \mathrm{I}_{T}^{1-\alpha} \psi(t)=0
$$

which lead to the desired result by taking a particular choice of $\psi$.

Theorem 4.2. The solution u of problem (3.1) is unique.

Proof. Let $u_{1}$ and $u_{2}$ be two weak solutions with the same initial condition. Let $w=u_{1}-u_{2}$. Then we have

$$
\left({ }_{0}^{C} D_{t}^{\alpha} w, v\right)_{g}+b_{g}\left(u_{1}, u_{2}, v\right)-b_{g}\left(u_{2}, u_{2}, v\right)+\nu((w, v))_{g}+\nu b_{g}\left(\frac{\nabla g}{g}, w, v\right)=0 .
$$

Taking $v=w$, one obtains

$$
\begin{aligned}
{ }_{0}^{C} D_{t}^{\alpha}|w|_{g}^{2}+2 \nu\|w\|_{g}^{2} & \leq 2 b_{g}\left(w, w, u_{2}\right)-2 \nu b_{g}\left(\frac{\nabla g}{g}, w, w\right) \\
& \leq c_{0}|w|_{g}\|w\|_{g}\left\|u_{2}\right\|_{g}+2 \nu \frac{|\nabla g|_{\infty}}{m_{0} \lambda^{1 / 2}}\|w\|_{g}^{2} \\
& \leq \nu\|w\|_{g}^{2}+c_{1}|w|_{g}^{2}\left\|u_{2}\right\|_{g}^{2}+2 \nu \frac{|\nabla g|_{\infty}}{m_{0} \lambda^{1 / 2}}\|w\|_{g}^{2} .
\end{aligned}
$$

It follows

$$
{ }_{0}^{C} D_{t}^{\alpha}|w|_{g}^{2}+\nu^{\prime}\|w\|_{g}^{2} \leq c_{1}|w|_{g}^{2}\left\|u_{2}\right\|_{g}^{2}
$$

where $\nu^{\prime}=\nu\left(1-\frac{2|\nabla g|_{\infty}}{m_{0} \lambda^{1 / 2}}\right)>0$. Hence

$$
{ }_{0}^{C} D_{t}^{\alpha}|w|_{g}^{2} \leq c_{1}|w|_{g}^{2}\left\|u_{2}\right\|_{g}^{2} .
$$

It follows

$$
|w(t)|_{g}^{2} \leq|w(0)|_{g}^{2}+\frac{c_{2}}{\Gamma(\alpha)} \int_{0}^{t}(t-s)^{\alpha-1}|w(s)|_{g}^{2}\left\|u_{2}(s)\right\|_{g}^{2} d s
$$


and by Gronwall inequality

$$
\begin{aligned}
|w(t)|_{g}^{2} & \leq|w(0)|_{g}^{2} \exp \left(\frac{c_{2}}{\Gamma(\alpha)} \int_{0}^{t}(t-s)^{\alpha-1}\left\|u_{2}(s)\right\|_{g}^{2} d s\right) \\
& \leq|w(0)|_{g}^{2} \exp \left(\frac{c_{2}}{\Gamma(\alpha)}\left(\left|u_{2}(0)\right|_{g}^{2}+\frac{1}{\nu^{\prime}} \int_{0}^{T}\|f(s)\|_{V_{g}^{\prime}}^{2 / \alpha_{1}}+\frac{T^{1+b}}{(1+b) \nu^{\prime}}\right)\right) .
\end{aligned}
$$

Since $|w(0)|_{g}=0$, it follows that $|w|_{g}=0$, which complete the proof.

\section{Optimal Control}

Let $\mathcal{U}_{g}=L^{2 / \alpha_{1}}\left(0, T ; H_{g}\right)$ be the space of controls. For every $f \in \mathcal{U}_{g}$, we denote by $S(f) \subset L^{2}\left(0, T ; V_{g}\right) \cap L^{\infty}\left(0, T ; H_{g}\right):=\mathcal{V}_{g}$ the solution set corresponding to $f$ of the problem (3.1). It is then clear, by definition, that $S(f)$ is nonempty for all $f \in \mathcal{U}_{g}$.

Theorem 5.1. Assume that $f_{n}, f \in L^{2 / \alpha_{1}}\left(0, T ; H_{g}\right)$ such that $f_{n}$ converges to $f$ weakly in $L^{2 / \alpha_{1}}\left(0, T ; H_{g}\right)$. Then, for every sequence $\left\{u_{n}\right\}_{n}$ such that $u_{n} \in S\left(f_{n}\right)$, we can find a subsequence of $\left\{u_{n}\right\}_{n}$ that converges weakly in $L^{2}\left(0, T, V_{g}\right)$ and weaklystar in $L^{\infty}\left(0, T ; H_{g}\right)$ to some $u \in L^{2}\left(0, T ; V_{g}\right) \cap L^{\infty}\left(0, T ; H_{g}\right)$ such that $u \in S(f)$.

Proof. Let $f_{n}, f \in L^{2 / \alpha_{1}}\left(0, T ; H_{g}\right)$ such that

$$
f_{n} \rightarrow f \text { weakly in } L^{2 / \alpha_{1}}\left(0, T ; H_{g}\right) \text {. }
$$

There exists $u_{n} \in L^{2}\left(0, T ; V_{g}\right) \cap L^{\infty}\left(0, T ; H_{g}\right)$ such that

$$
\begin{aligned}
{ }_{0}^{C} D_{t}^{\alpha} u_{n}(t)+\nu A_{g} & u_{n}(t)+\nu C_{g} u_{n}(t) \\
& +B_{g}\left(u_{n}(t), u_{n}(t)\right)=f_{n}(t) \quad \text { for a.e. } t \in[0, T] .
\end{aligned}
$$

Multiplying by $u_{n}$, one obtains

$$
{ }_{0}^{C} D_{t}^{\alpha}\left|u_{n}(t)\right|_{g}^{2}+\nu^{\prime}\left\|u_{n}(t)\right\|_{g}^{2} \leq \frac{1}{\nu}\left\|f_{n}(t)\right\|_{g^{\prime}}^{2} .
$$

This yields to

$$
\begin{aligned}
|u(t)|_{g}^{2}+\nu^{\prime} \int_{0}^{t}(t-s)^{\alpha-1}\|u(s)\|_{g}^{2} d s & \leq|u(0)|_{g}^{2}+\frac{1}{\nu} \int_{0}^{t}(t-s)^{\alpha-1}\left\|f_{n}(s)\right\|_{g^{\prime}}^{2} d s \\
& \leq|u(0)|_{g}^{2}+\frac{1}{\nu} \int_{0}^{T}\left\|f_{n}(s)\right\|_{g^{\prime}}^{\frac{2}{\alpha_{1}}} d s+\frac{T^{1+b}}{(1+b) \nu} .
\end{aligned}
$$

Therefore the sequence $\left\{u_{n}\right\}_{n}$ is bounded in $L^{2}\left(0, T ; V_{g}\right)$ and in $L^{\infty}\left(0, T ; H_{g}\right)$. Thus there exists an element $\hat{u} \in L^{2}\left(0, T ; V_{g}\right) \cap L^{\infty}\left(0, T ; H_{g}\right)$ such that $\left\{u_{n}\right\}_{n}$ converges weakly in $L^{2}\left(0, T ; V_{g}\right)$ and star-weakly in $L^{\infty}\left(0, T ; H_{g}\right)$. Moreover, with the same argument as in the existence theorem, the sequence $\left\{u_{n}\right\}_{n}$ converges to $\hat{u}$ strongly in $L^{2}\left(0, T ; H_{g}\right)$. Finally, from the continuity of $A_{g}$ and $C_{g}$ and the weak continuity of $B_{g}$ we deduce that $\hat{u} \in S(f)$.

Corollary 5.2. Assume that $\varphi_{n}, \varphi, h \in L^{2 / \alpha_{1}}\left(0, T ; H_{g}\right)$ such that $\varphi_{n}$ converges to $\varphi$ weakly in $L^{2 / \alpha_{1}}\left(0, T ; H_{g}\right)$. Then, for every sequence $\left\{u_{n}\right\}_{n}$ such that $u_{n} \in$ $S\left(\varphi_{n}+h\right)$, we can find a subsequence of $\left\{u_{n}\right\}_{n}$ that converges weakly in $L^{2}\left(0, T, V_{g}\right)$ 
and weakly-star in $L^{\infty}\left(0, T ; H_{g}\right)$ to some $u \in L^{2}\left(0, T ; V_{g}\right) \cap L^{\infty}\left(0, T ; H_{g}\right)$ such that $u \in S(\varphi+h)$.

Proof. It suffices to take $f_{n}=\varphi_{n}+f$ and apply Theorem 5.1.

Let $\mathcal{U}_{g}$ be a real Hilbert space and $C \in \mathscr{L}\left(\mathcal{U}_{g}, H_{g}\right)$ a bounded linear operator from $\mathcal{U}_{g}$ to $H_{g}$. We have the following corollary:

Corollary 5.3. Assume that $w_{n}, w \in L^{2 / \alpha_{1}}\left(0, T ; \mathcal{U}_{g}\right)$ and $f \in L^{2 / \alpha_{1}}\left(0, T ; V_{g}^{\prime}\right)$ such that $w_{n}$ converges to $w$ weakly in $L^{2 / \alpha_{1}}\left(0, T ; \mathcal{U}_{g}\right)$. Then, for every sequence $\left\{u_{n}\right\}_{n}$ such that $u_{n} \in S\left(C w_{n}+f\right)$, we can find a subsequence of $\left\{u_{n}\right\}_{n}$ that converges weakly in $L^{2}\left(0, T, V_{g}\right)$ and weakly-star in $L^{\infty}\left(0, T ; H_{g}\right)$ to some $u \in L^{2}\left(0, T ; V_{g}\right) \cap$ $L^{\infty}\left(0, T ; H_{g}\right)$ such that $u \in S(C w+f)$.

Proof. It suffices to take $f_{n}=C w_{n}$ and apply Corollary 5.2 .

Let $\mathcal{U}_{\text {ad }}$ be a nonempty subset of $\mathcal{U}_{g}$ consisting of admissible controls. Let $\mathscr{F}$ : $\mathcal{U}_{g} \times \mathcal{V}_{g} \rightarrow \mathbb{R}$ be the objective functional we want to minimize. The control problem reads as follows: Find a control $\hat{f} \in \mathcal{U}_{a d}$ and a state $\hat{u} \in S(\hat{f})$ such that

$$
\mathscr{F}(\hat{f}, \hat{u})=\inf \left\{\mathscr{F}(f, u): f \in \mathcal{U}_{a d}, u \in S(f)\right\} .
$$

A couple which solves (5.1) is called an optimal solution. The existence of such optimal control can be proved by using Theorem5.1. To do so, we need the following additional hypotheses:

$H\left(\mathcal{U}_{a d}\right) \quad \mathcal{U}_{a d}$ is a bounded and weakly closed subset of $\mathcal{U}$.

$H(\mathscr{F}) \quad \mathscr{F}$ is lower semicontinuous with respect to $\mathcal{U} \times \mathcal{V}$ endowed with the weak topology.

Theorem 5.4. Assume that $H(g), H\left(\mathcal{U}_{a d}\right)$ and $H(\mathscr{F})$ are fulfilled. Then the problem (5.1) has an optimal control.

Proof. Let $\left(f_{n}, u_{n}\right)$ be a minimizing sequence for the problem (5.1), i.e $f_{n} \in \mathcal{U}_{a d}$ and $u_{n} \in S\left(f_{n}\right)$ such that

$$
\lim _{n \rightarrow \infty} \mathscr{F}\left(f_{n}, u_{n}\right)=\inf \left\{\mathscr{F}(f, u): f \in \mathcal{U}_{a d}, u \in S(f)\right\}=: m .
$$

It follows that the sequence $f_{n}$ belongs to a bounded subset of the reflexive Banach space $L^{2 / \alpha_{1}}\left(0, T ; V_{g}\right)$. We may then assume that $f_{n} \rightarrow \hat{f}$ weakly in $L^{2 / \alpha_{1}}\left(0, T ; V_{g}^{\prime}\right)$ (by passing to a subsequence if necessary). By $H\left(\mathcal{U}_{a d}\right)$, we have $\hat{f} \in \mathcal{U}_{a d}$. From Theorem 5.1, we obtain, by again passing to a subsequence if necessary, that $u_{n} \rightarrow \hat{u}$ weakly in $L^{2}\left(0, T ; V_{g}\right)$ and star-weakly in $L^{\infty}\left(0, T ; H_{g}\right)$ with $\hat{u} \in S(\hat{f})$. By $H(\mathscr{F})$, we have $m \leq \mathscr{F}(\hat{f}, \hat{u}) \leq \liminf _{n \rightarrow \infty} \mathscr{F}\left(f_{n}, u_{n}\right)=m$. Which completes the proof.

Let $f \in L^{2 / \alpha_{1}}\left(0, T ; V_{g}^{\prime}\right)$ and $z \in L^{\frac{2}{\alpha_{1}}}\left(0, T ; H_{g}\right)$. Consider the following optimal problem $(P)$ :

$$
\text { Minimize } J(u, w)=\frac{1}{2} \int_{0}^{T} \int_{\Omega}(u(t, x)-u(s, x))^{2} g(x) d x d t+\int_{0}^{T} h(w(t)) d t
$$

over $(u, w) \in\left(L^{2}\left(0, T ; V_{g}\right) \cap L^{\infty}\left(0, T ; H_{g}\right)\right) \times L^{2 / \alpha_{1}}\left(0, T ; U_{g}\right)$ subject to $u \in S(C w+$ $f)$, i.e. $u$ satisfies 
We assume that:

$$
\left\{\begin{aligned}
{ }_{0}^{C} D_{t}^{\alpha} u+\nu A_{g} u+\nu C_{g} u+B_{g}(u, u) & = & C w+f \\
u(0) & = & u_{0} .
\end{aligned}\right.
$$

$H(h) \quad$ The function $h: U_{g} \rightarrow \mathbb{R}$ is convex, lower semicontinuous and satisfies

$$
|h(w)| \geq b_{1}|w|_{U_{g}}^{\frac{2}{\alpha_{1}}}+b_{2}
$$

for some $b_{1}>0, b_{2} \in \mathbb{R}$.

Theorem 5.5. Assume $H(g)$ and $H(h)$. Then problem $(P)$ has at least one solution $(\hat{u}, \hat{w}) \in\left(L^{2}\left(0, T ; V_{g}\right) \cap L^{\infty}\left(0, T ; H_{g}\right)\right) \times L^{2 / \alpha_{1}}\left(0, T ; U_{g}\right)$.

Proof. Let $\left(u_{n}, w_{n}\right)$ be a minimizing sequence of $(P)$. By $H(h),\left(w_{n}\right)_{n}$ is bounded in $L^{2 / \alpha_{1}}\left(0, T ; U_{g}\right)$. Hence, there exists a subsequence converging weakly in $L^{2 / \alpha_{1}}\left(0, T ; U_{g}\right)$ to some $w$. It follows by Corollary 5.3 that $u_{n}$ converges weakly in $L^{2}\left(0, T ; V_{g}\right)$ and weakly-star in $L^{\infty}\left(0, T ; H_{g}\right)$ to some $u \in S(C w+f)$. Take $\mathcal{U}_{a d}=L^{2 / \alpha_{1}}(0, T ; \operatorname{Im} C)$ and note that $J(u, w)=\mathscr{F}(C w+f, u)$. It follows by Theorem [5.4, that problem $(P)$ has at least one solution as required.

Acknowledgments. We would like to express our gratitude to the Editor for taking time to handle the manuscript and to anonymous referees whose constructive comments are very helpful for improving the quality of our paper.

\section{REFERENCES}

[1] O.P. Agrawal, Fractional variational calculus in terms of Riesz fractional derivatives, J. Phys. A 40 (2007) 6287-6303.

[2] A.A. Alikhanov, A priori estimates for solutions of boundary value problems for fractionalorder equations, Differ. Equ. 46 (2010) 660-666.

[3] C. T. Anh and D. T. Quyet, $g$-Navier-Stokes equations with infinite delays, Vietnam J. Math. 40 (2012), no. 1, 57-78.

[4] C. T. Anh and D. T. Quyet, Long-time behavior for $2 D$ non-autonomous $g$-Navier-Stokes equations, Ann. Polon. Math. 103 (2012), no. 3, 277-302.

[5] C. T. Anh, N. V. Thanh and N. V. Tuan, On the stability of solutions to stochastic $2 D$ $g$-Navier-Stokes equations with finite delays, Random Oper. Stoch. Equ., Volume 25, Issue 4, Pages 211-224 2017.

[6] Bae, H., Roh, J.,Existence of Solutions of the g-Navier-Stokes Equations, Taiwanese J. Math., 8, No. 1, 85-102, 2004.

[7] R. Camassa, D.D. Holm, C.D. Levermore, Long-time effects of bottom topograpy in shallow water, Physica D. 98 (2-4) (1996) 258-286.

[8] G.P. Galdi, An Introduction to the Mathematical Theory of the Navier-Stokes Equations: Steady-State Problems, Springer Science and Business Media, 2011.

[9] J.K. Hale, G. Raugel, Reaction-diffusion equation on thin domains, J. Math. Pures Appl. 71 (1) (1992) 33-95.

[10] J.K. Hale, G. Raugel, A damped hyperbolic equation on thin domains, Trans. Amer. Math. Soc. 329 (1) (1992) 185-219.

[11] Iftimie, D., The 3D Navier-Stokes equations seen as a perturbation of the $2 D$ Navier-Stokes equations, Bull. Soc. Math., France, 127, 473-517, 1999.

[12] J.P. Jiang, Y.R. Hou and X.X. Wang, Pullback attractor of $2 D$ nonautonomous $g$-NavierStokes equations with linear dampness, Appl. Math. Mech. (English Ed.) 32 (2011), no. 2, $151-166$ 
[13] J. Jiang and Y. Hou, The global attractor of $g$-Navier-Stokes equations with linear dampness on R2, Appl. Math. Comput. 215 (2009), no. 3, 1068-1076

[14] J.P. Jiang and Y.R. Hou, Pullback attractor of 2D non-autonomous $g$-Navier-Stokes equations on some bounded domains, Appl. Math. Mech. (English Ed.) 31 (2010), no. 6, 697-708.

[15] J.P. Jiang and X.X. Wang, Global attractor of $2 D$ autonomous $g$-Navier-Stokes equations, Appl. Math. Mech. (English Ed.) 34 (2013), no. 3, 385-394.

[16] H. Kwean, The $H^{1}$-compact global attractor of two-dimensional $g$-Navier-Stokes equations, Far East J. Dyn. Syst. 18 (2012), no. 1, 1-20.

[17] H. Kwean and J. Roh, The global attractor of the 2D $g$-Navier-Stokes equations on some unbounded domains, Commun. Korean Math. Soc. 20 (2005), no. 4, 731-749.

[18] M. Kwak, H. Kwean and J. Roh, The dimension of attractor of the $2 D g$-Navier-Stokes equations, J. Math. Anal. Appl. 315 (2006), no. 2, 436-461.

[19] A.A. Kilbas, H.M. Srivastava, J.J. Trujillo, Theory and Applications of Fractional Differential Equations, in: North-Holland Math. Stud., vol. 204, Elsevier Science B.V, Amsterdam, 2006.

[20] P.G. Lemarié-Rieusset, Recent Developments in the Navier-Stokes Problem, Chapman CRC Press, 2002.

[21] C.D. Levermore, M. Oliver, E.S. Titi, Global well-posedness for models of shallow water in a basin with varrying bottom, Indiana Univ. Math. J. 45 (2) (1996) 479-510.

[22] C.D. Levermore, M. Oliver, E.S Titi, Global well-posedness for the lake equations, Physica D 98 (1996) 492-596.

[23] J.L. Lions, Sur l'existence de solutions des équations de Navier-Stokes, C. R. Acad. Sci., Paris 248 (1959) 2847-2849.

[24] G. Lukaszewicz, P. Kalita, Navier-Stokes Equations: An Introduction with Applications, Springer, 2016

[25] H. Mahdioui, S. Ben Aadi, K. Akhlil, Hemivariational Inequality for Navier-Stokes Equations: Existence, Dependence and Optimal Control. https://arxiv.org/abs/2001.09449 (In presse).

[26] D. T. Quyet, Asymptotic behavior of strong solutions to $2 D g$-Navier-Stokes equations, Commun. Korean Math. Soc. 29 (2014), no. 4, 505-518.

[27] D. T. Quyet, Pullback attractors for strong solutions of $2 D$ non-autonomous $g$-Navier-Stokes equations, Acta Math. Vietnam. 40 (2015), no. 4, 637-651.

[28] D. T. Quyet, Pullback attractors for 2D $g$-Navier-Stokes equations with infinite delays, Commun. Korean Math. Soc. 31 (2016), no. 3, 519-532.

[29] D. T. Quyet and N. V. Tuan, On the stationary solutions to 2D $g$-Navier-Stokes equations, Acta Math. Vietnam. 42 (2017), no. 2, 357-367.

[30] G Raugel, G.R. Sell, Navier stokes equations on thin 3D Domains I. Global attractors and global regularity of solutions, J.Amer.Math.Soc. 6 (3) (1993) 503-568.

[31] Roh, J., g-Navier-Stokes equations, Thesis, University of Minnesota, 2001.

[32] Roh, J., Dynamics of the g-Navier-Stokes equations, J. Differ. Equ., 211, No. 2, 452-484, 2005.

[33] Roh, J., Geometry of $L^{2}(\Omega ; g)$, J. Chungcheong Math. Soc., 19, No.3, 283-289, 2006.

[34] Tachim Medjo, T. (2019). A note on the regularity of weak solutions to the coupled 2D Allen-Cahn-Navier-Stokes system, Journal of Applied Analysis, 25(1), 111-117.

[35] Temam, R., Navier-Stokes Equations, Theory and Numerical Analysis, vol. 2 of Studies in Mathematics and Its Applications, North-Holland, Amsterdam, The Netherlands, 3rd edition, 1984.

[36] D. Wu, The finite-dimensional uniform attractors for the nonautonomous $g$-Navier-Stokes equations, J. Appl. Math. 2009 (2009), Article ID 150420.

[37] Wu, D., On the Dimension of the Pullback Attractors for g-Navier-Stokes Equations, Discrete Dyn. Nature Soc., 2010, Article ID 893240, 16 pages.

[38] Zaja czkowski, W. M. (1998). On Nonstationary Motion of a Compressible Barotropic Viscous Fluid with Boundary Slip Condition, Journal of Applied Analysis, 4(2), 167-204

[39] Y. Zhou, L. Peng, Weak solutions of the time-fractional Navier-Stokes equations and optimal control, Volume 73, Issue 6, 1016-1027, 2017.

[40] Y. Zhou, Basic Theory of Fractional Differential Equations, World Scientific, Singapore, 2014. 
[41] Y. Zhou, Fractional Evolution Equations and Inclusions: Analysis and Control, Academic Press, 2016.

Plydisciplinary Faculty of Ouarzazate

SULTANA.BENAADI@EDU.UIZ.AC.MA

K.AKHLIL@UIZ.AC.MA

KHADIJA.AAYADI@GMAIL.COM 